\title{
Robust Fault-Tolerant Control of In-Wheel Driven Bus with Cornering Energy Minimization
}

\author{
András Mihály 1 - Péter Gáspár1,2 - Balázs Németh ${ }^{1,2}$ \\ 1Budapest University of Technology and Economics, Department of Control for Transportation and Vehicle Systems, Hungary \\ 2Hungarian Academy of Sciences, Institute for Computer Science and Control, Hungary
}

The aim of this paper is to design fault-tolerant and energy optimal trajectory tracking control for a four-wheel independently actuated (FWIA) electric bus with a steer-by-wire steering system. During normal driving conditions, the architecture of the proposed controller enables the bus to select an energy optimal split between steering intervention and torque vectoring, realized by the independently actuated in-wheel motors by minimizing the cornering resistance of the bus. In the case of skidding or a fault event of an in-wheel motor or the steering system, a high-level control reconfiguration using linear parameter varying (LPV) techniques is applied to reallocate control signals in order to stabilize the bus. The main novelty of the paper is the control reconfiguration method based on the specific characteristics of the in-wheel bus which enables introducing such scheduling variables, with which the safety and efficiency of the FWIA bus can be enhanced.

Keywords: In-wheel motor, FWIA bus, reconfigurable control, cornering resistance

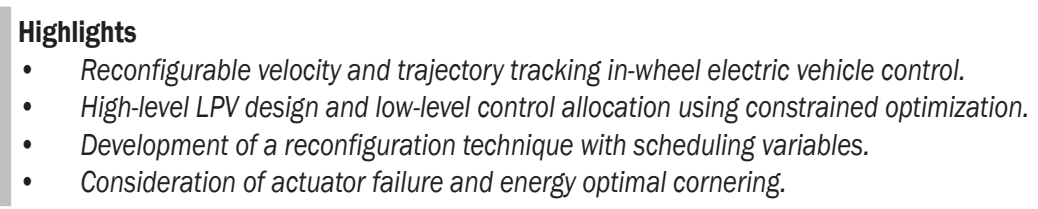

\section{INTRODUCTION}

As economical and environment-friendly hybrid/ electric vehicles become increasingly popular, researchers and automotive companies also focus on the development of in-wheel electric vehicles, which have several benefits in comparison to conventional vehicles. From a vehicle dynamic point of view, the fast and precise torque generation of the hub motors lends torque vectoring capability to the vehicle with which manoeuvrability can be enhanced significantly [1] and [2]. The electric in-wheel motors must also be integrated with friction brakes, as proposed in [3]. By knowing the characteristics of the in-wheel engines and the hydraulic brake system, energy optimal torque distribution can be achieved as proposed by [4] and [5]. Moreover, high-efficiency regenerative braking can be implemented [6].

The capabilities of a four-wheel independently actuated (FWIA) vehicle enable novel techniques to improve safety and economy. Recently, a sliding mode control has been developed by [7] to deal with a faulty in-wheel motor by rearranging steering geometry depending on the location of the fault. Furthermore, the steer-by-wire steering system failures can be handled more effectively in an FWIA vehicle by using differential drive-assisted steering, as presented in [8]. The performance degradation of both the steering system and the in-wheel motors were addressed in a more general manner in [9]. The aim of the paper [10] was to design a trajectory and velocity tracking reconfiguration control method for FWIA vehicle, in which both types of actuator failures and cornering resistance minimization are handled simultaneously in order to enhance the economic performance of the vehicle.

The novelty of the proposed method in this paper lies in the high-level linear parameter varying (LPV) control reconfiguration strategy based on the specific design of weighting functions' handling actuator selection. The method enables the vehicle to dynamically modify the prescribed control values best suited for the actual vehicle state and the corresponding priority regarding safety and economy.

The paper is organized as follows: Section 1 introduces the vehicle model used for the control of the FWIA bus. Section 2 presents the control design found on an LPV framework and the structure of the fault tolerant and energy efficient control reconfiguration. Section 3 defines the allocation of the high-level control signals based on vehicle dynamics. Section 4 demonstrates the effect of the introduced method in a software-in-the-loop (SIL) simulation environment. Finally, some concluding statements are listed in Section 5.

*Corr. Author's Address: Budapest University of Technology and Economics Department of Control for Transportation and Vehicle Systems,

H-1111 2. Stoczek street, Budapest, Hungary, mihaly.andras@mail.bme.hu 


\section{CONTROL-ORIENTED MODEL OF THE BUS}

The goal of the design is to ensure trajectory and velocity tracking for the FWIA bus, taking longitudinal and lateral dynamics into account while neglecting the vertical behaviour of the bus. Thus, for the modelling of the FWIA bus dynamics, the well-known twowheeled bicycle model is used, see Fig. 1.

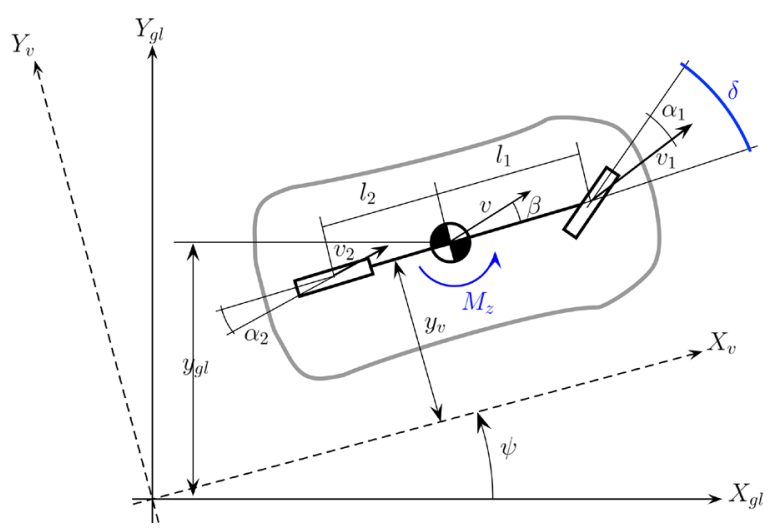

Fig. 1. Single track bicycle model

The motion equations in the planar plane can be written as follows:

$$
\begin{gathered}
J \ddot{\psi}=l_{1} F_{y f}-l_{2} F_{y r}+M_{z}, \\
m \dot{\xi}(\dot{\psi}+\dot{\beta})=F_{y f}+F_{y r}, \\
m \ddot{\xi}=F_{l}-F_{d},
\end{gathered}
$$

where $m$ is the bus mass, $J$ is the yaw inertia, $F_{y f}=c_{1} \alpha_{1}$ and $F_{y r}=c_{2} \alpha_{2}$ are the lateral tyre forces on the front and rear axle, $c_{1}$ and $c_{2}$ are the tyres' cornering stiffness. The distances measured from the centre of gravity to the front and rear axles are denoted by $l_{1}$ and $l_{2}$. The side slip angles of the front and rear wheels are $\alpha_{1}=\delta-\beta-\dot{\psi} l_{1} / \dot{\xi}$ and $\alpha_{2}=-\beta+\dot{\psi} l_{2} / \dot{\xi}$. The yaw rate of the bus is denoted with $\dot{\psi}$, the bus side-slip angle is $\beta$ and $\xi$ is the longitudinal displacement of the FWIA bus.

The high-level control inputs of the bus are the longitudinal force noted with $F_{l}$, the yaw moment $M_{z}$ generated by torque vectoring, and the steering angle $\delta$ of the front wheels. The disturbance force $F_{d}$ contains the drag, rolling and road slope disturbances: $F_{d}=F_{d 1}+F_{d 2}+F_{d 3}$. Here, the aerodynamic drag is a function of the drag coefficient $c_{w}$, the air density $\rho_{\text {aero }}$, the frontal area size $A_{F}$ and the velocity of the bus: $F_{d 1}=0.5 c_{w} \rho_{\text {aero }} A_{F} \dot{\xi}^{2}$. The rolling resistance force depends on vehicle mass $m$, rolling resistance coefficient $f$ and road slope angle $\theta: F_{d 2}=m g f \cos \theta$, where $g$ is the gravitational constant. Finally, the road slope disturbance is calculated as: $F_{d 3}=m g \sin \theta$.

Since the nonlinearity of the system described by the differential equations of Eq. (1) is caused by the velocity $\dot{\xi}$ of the bus, choosing it as a scheduling variable $\rho_{1}=\dot{\xi}$ the nonlinear model is rewritten as an LPV model. Note that another scheduling variable $\rho_{2}$ is also introduced responsible for the high-level control allocation between steering and yaw moment generation. The main goal of the proposed method presented in the paper is to determine the value of $\rho_{2}$ in such way that the energy efficiency and safety of the FWIA bus can be guaranteed. Thus, a real-time calculation is carried out to define $\rho_{2}^{M_{z}}$ and $\rho_{2}^{\delta}$ related to safety and $\rho_{2}^{e}$ related to energy efficiency, while simple decision logic sets the value of $\rho_{2}$ considering priorities.

The state-space representation of the LPV model can be written as follows:

$$
\dot{\bar{x}}=A\left(\rho_{1}\right) \bar{x}+B_{1} \bar{w}+B_{2} \bar{u},
$$

where the state vector $\bar{x}=\left[\begin{array}{llll}\dot{\xi} & \xi & \dot{\psi} & \beta\end{array}\right]^{T}$ contains the bus velocity, the displacement, the yaw rate and the side-slip angle. Note that the input vector $\bar{u}=\left[\begin{array}{lll}F_{l} & \delta & M_{z}\end{array}\right]^{T}$ contains the high-level control inputs, the output vector $\bar{y}=\left[\begin{array}{ll}\dot{\xi} & \dot{\psi}\end{array}\right]^{T}$ contains the measured velocity and the yaw rate, while the disturbance vector is $\bar{w}=\left[F_{d}\right]^{T}$.

\section{DESIGN OF ENERGY OPTIMAL AND FAULT TOLERANT CONTROLLER}

For the nonlinear model of the FWIA bus, a gain scheduling LPV controller is necessary to guarantee a global solution. The reference signals for the bus to follow are the reference velocity and the yaw rate. The former is set by the driver, while the latter is also given by the driver steering intervention $\delta_{d}$ as follows [11]: $\quad \dot{\psi}_{\text {ref }}=\dot{\xi} / d \cdot e^{-t / \tau} \cdot \delta_{d}$, where $\tau$ is the time constant, $d$ is a parameter depending on the bus geometry and velocity. With $\dot{\xi}_{\text {ref }}$ denoting the reference velocity set by the driver, the two signals are put in a reference vector $\bar{R}=\left[\dot{\xi}_{\text {ref }} \dot{\psi}_{\text {ref }}\right]^{T}$. Thus, the control task is to follow the signals given in vector $\bar{R}$, for which two optimization criteria must be satisfied at the same time. Both the velocity error $z_{\dot{\xi}}=\left|\dot{\xi}_{\text {ref }}-\dot{\xi}\right|$ and yaw rate error $z_{\dot{\psi}}=\left|\dot{\psi}_{\text {ref }}-\dot{\psi}\right|$ must be minimized with the consideration of the optimization criterion $z_{\xi} \rightarrow 0$ and $z_{\dot{\psi}} \rightarrow 0$. These are represented with the performance vector $\bar{z}_{1}=\left[\begin{array}{ll}z_{\dot{\xi}} & z_{\dot{\psi}}\end{array}\right]^{T}$, while the limitation of the control inputs connected to the physical set-up of the in-wheel 
motors and steering system are defined by a second performance vector $\bar{z}_{2}=\left[\begin{array}{lll}F_{l} & \delta & M_{z}\end{array}\right]^{T}$.

\subsection{High-Level Controller Design}

The proposed high-level controller is based on the closed-loop $P-K-\Delta$ architecture, as depicted in Fig. 2. Here, $P$ is the augmented plant in which uncertainties given by $\Delta$ are taken into consideration and $K$ is the controller.

The design is based on selecting relevant weighting functions representing control objectives, disturbances, and sensor noises. Weighting functions $W_{p}$ ensure the accommodation of performances defined in $\bar{z}_{1}$, serving as penalty functions. Disturbances and sensor noises are considered with $W_{w}$ and $W_{n}$, while the neglected dynamics of the bus are represented by $W_{u}$.

The focus of the paper is connected to the weighting function $W_{a}$, responsible for the control reconfiguration between actuators. The goal of the design is to ensure an optimal split between steering and torque vectoring for the bus, considering both the efficiency and safety of the FWIA bus. These criteria are realized by the scheduling parameter $\rho_{2} \in[0.01,1]$, which holds for the scaling of the actuators. Thus, the weighting function of the steering $W_{a \delta}=\left(\delta_{\max } \chi_{1}\right) /\left(\rho_{2}\right)$ and the differential torque generation $W_{a M z}=\left(\rho_{2}\right) /\left(M_{z \max } \chi_{2}\right)$ are dependent on $\rho_{2}$, with $\delta_{\max }$ and $M_{z \max }$ representing the maximal steering angle and yaw moment, $\chi_{1}$ and $\chi_{2}$ are design parameters tuned to achieve an appropriate control reallocation.

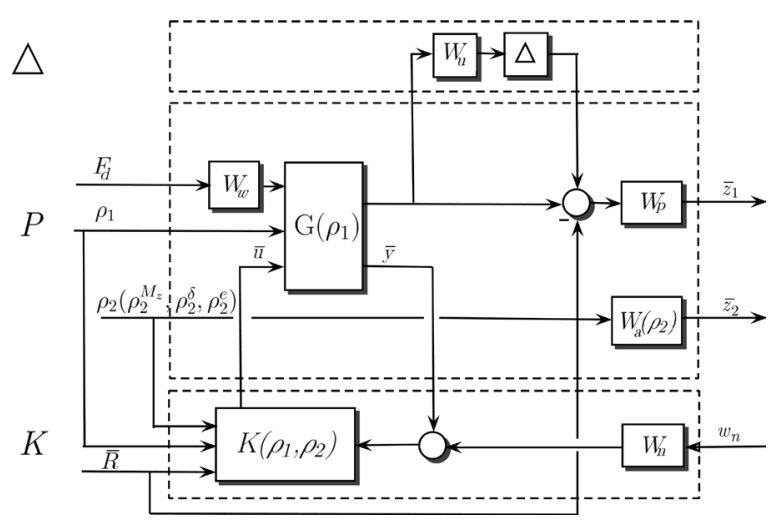

Fig. 2. Closed-loop structure

The consideration of safety and efficiency described later in Section 2.2 and Section 2.3 are implemented by the defining the value of $\rho_{2}$ based on the calculated parameters $\rho_{2}^{M_{z}}, \rho_{2}^{\delta}$ and $\rho_{2}^{e}$. The minimization task for the LPV performance problem already described in [12] is written as follows:

$$
\inf _{K} \sup _{\rho \in F_{\mathrm{F}}\|w\|_{2} \neq 0, w \in L_{2}} \sup _{2} \frac{\|z\|_{2}}{\|w\|_{2}} \leq \gamma .
$$

The goal of the design is to select a parametervarying controller in order to guarantee quadratic stability, while the induced $L_{2}$ norm from the disturbance $\bar{w}$ to the performances $\bar{z}$ remains smaller than $\gamma$. The $L P V$ control synthesis is detailed in [13].

\subsection{Consideration of Fault Events and Wheel Slip}

The reconfiguration method in the case of a faulty in-wheel electric motor or the skidding of one of the wheel is based on the specific property of the in-wheel motor construction. The fast and accurate torque generation of the electric motors and the direct link to the wheels enables a precise estimation of the transmitted longitudinal wheel forces for each wheel of the FWIA bus. For this purpose, the wheel dynamics must be considered, given as follows:

$$
J_{i j} \dot{\omega}_{i j}=T_{i j}^{\text {motor }}-R_{e f f} F_{i j}^{\text {trans }},
$$

where $\quad J_{i j}, \quad i \in[f=$ front, $r=$ rear $]$, $j \in[L=l e f t, R=r i g h t]$ is the wheel inertia, $\dot{\omega}_{i j}$ is the measured angular acceleration, $R_{\text {eff }}$ is the effective rolling radius of the wheels, $T_{i j}^{\text {motor }}$ is the torque produced by the in-wheel engines, which can be measured. Thus, assuming no wheel slip the transmitted longitudinal drive or brake force $F_{i j}^{\text {trans }}$ can be calculated. When longitudinal wheel slip $\lambda$ occurs, the friction force $F_{i j}^{\text {trans }}$ can still be estimated as described in [14] by considering the relation of the friction coefficient and wheel slip $\mu-\lambda$ given by the Pacejka Magic Formula, see [15].

Thus, by using the estimated transmitted torque of each wheel $T_{i j}^{\text {trans }}=R_{e f f} F_{i j}^{\text {trans }}$ and assuming a small steering angle $\delta$ of the front wheels the transmitted yaw moment of the in-wheel bus can be given as follows:

$$
M_{z}^{\text {trans }}=\frac{T_{f}^{\text {trans }}}{R_{\text {eff }}} \frac{b_{f}}{2}+\frac{T_{r}^{\text {trans }}}{R_{\text {eff }}} \frac{b_{r}}{2}
$$

where $T_{f}^{\text {trans }}=-T_{f L}^{\text {trans }}+T_{f R}^{\text {trans }}$ and $T_{r}^{\text {trans }}=-T_{r L}^{\text {trans }}+T_{r R}^{\text {trans }}$ are the transmitted torque at the front and rear concerning $M_{z}^{\text {trans }}$, moreover, $b_{f}$ and $b_{r}$ are the front and rear track.

Hence, the relation of the desired yaw moment given by the high-level controller $M_{z}$ and the achieved 
yaw moment $M_{z}^{\text {trans }}$ of the FWIA bus serves as the indicator for the bus dynamic state related to the trajectory-tracking task. For example, when the transmitted yaw moment $M_{z}^{\text {trans }}$ becomes significantly smaller than the prescribed value $M_{z}$, the bus is skidding, or an internal failure has occurred in one of the electric motors. Thus, the cornering manoeuvre can only be evaluated by using more steering and less yaw moment; therefore, the negative effect of a wheel slip or motor failure can be eliminated. Hence, one of the aims of the reconfiguration presented in the paper is to reallocate the high-level control signals in such cases, by introducing the variable $\rho_{2}^{M_{z}}$ as follows:

$$
\rho_{2}^{M_{z}}=\left|\frac{\left|M_{z}-M_{z}^{\text {trans }}\right|}{M_{z}}\right| \text {. }
$$

Accordingly, if the bus can transmit the prescribed yaw moment during a cornering manoeuvre, the value of $\rho_{2}^{M_{z}}$ remains small and the value of $\rho_{2}$ responsible for the high-level control allocation is that set by the result of the energy optimization method detailed later in Section 2.3 or the value of $\rho_{2}^{\delta}$ due to a steering system failure. In the case of a faulty electric motor or wheel slip due to critical bus dynamics, the value of $\rho_{2}^{M_{z}}$ increases and the energy optimal split between steering and yaw moment generation is overwritten by the safety critical distribution with altering the value of $\rho_{2}$ to be equal with $\rho_{2}^{M_{z}}$. Thus, with modification of the weighting function $W_{a}$ of the LPV controller, steering intervention becomes more pronounced to overcome the effect of lateral dynamic performance degradation due to faulty electric motor or wheel spin.

Fault-tolerant control methods for steer-by-wire steering systems have already been presented by researchers. Although faulty steering occurs rarely in comparison to the performance degradation or wheel slip related to the in-wheel motors, this present paper also deals with such event to guarantee bus stability. Here, the aim of the fault tolerant design is to substitute the effect of the steering in case of a fault event by reconfiguring the high-level controller, with which additional differential torque is generated by the in-wheel motors. The fault of the steering is assumed to be detected by FDI, as proposed by [16].

When the fault is detected, the scheduling variable $\rho_{2}^{\delta}=0$ is applied overwriting the value of the actual $\rho_{2}$. Thus, weighting function $W_{a}$ of the LPV controller is modified in such a way, that the highlevel controller prescribes solely yaw moment signal for the FWIA bus. Thus, in the case of a steering system failure, the cornering manoeuvres are evaluated using only the precise torque vectoring ability of the in-wheel bus.

\subsection{Consideration of Cornering Resistance}

The efficiency of the FWIA bus can be enhanced by optimizing the high-level control inputs of the bus, i.e. the steering angle $\delta$ and yaw moment $M_{z}$. The aim of this procedure is to minimize cornering forces, i.e. the longitudinal disturbances affecting the bus related to the cornering manoeuvre. Using the two-wheeled bicycle model, the cornering force $F_{c}$ is calculated, omitting forces related to the drag, road slope and rolling resistance of the wheels as follows:

$$
F_{c}=2 F_{y f} \sin \left(\alpha_{1}+\delta\right)+2 F_{y r} \sin \left(\alpha_{2}\right)
$$

Thus, during a cornering manoeuvre the power loss of the bus related to the cornering is $P=F_{c} \dot{\xi}+M_{z} \dot{\psi}$. Assuming no energy recuperation, the cornering energy of the FWIA bus can simply be calculated. Using small angle approximation $\sin \left(\alpha_{1}\right) \approx \alpha_{1}$ and $\sin \left(\alpha_{2}\right) \approx \alpha_{2}$, the following formula can be derived: $P=\left(2 c_{1} \alpha_{1}\left(\alpha_{1}+\delta\right)+2 c_{2}\left(\alpha_{2}\right)^{2}\right) \dot{\xi}+M_{z} \dot{\psi}$. Ignoring $\alpha_{1}^{2}$ and $\alpha_{2}^{2}$ given their small values, the power loss related to cornering can finally be expressed as follows, see [17]:

$$
P=\left(2 c_{1} \alpha_{1} \delta\right) \dot{\xi}+M_{z} \dot{\psi} .
$$

Note that in the calculation of the power loss $\alpha_{1}=\delta-\beta-\dot{\psi} l_{1} / \dot{\xi}$ need to be known. This requires the knowledge of the bus side slip angle $\beta$, which can be estimated with different methods, as proposed by [18].

The role of the control allocation in the power loss due to cornering is well represented, as the power loss is a function of several bus dynamic states and the values of the control inputs $\delta$ and $M_{z}$. Thus, the objective of the cornering resistance minimization task is to create an optimal steering angle $\delta^{e}$ and yawmoment $M_{z}^{e}$, with which the power loss expressed in Eq. (8) can be minimized. Note, that the total yaw moment induced by the steering intervention and differential torque generation must be unchanged. Thus, using the torque Eq. (1) and assuming the steady state of the bus dynamic parameters $(\beta, \dot{\psi}$ and $\dot{\xi})$, the following constraint can be defined:

$$
\left(c_{1} l_{1} \delta^{e}+M_{z}^{e}\right)-\left(c_{1} l_{1} \delta+M_{z}\right)=0 .
$$

Since the steer-by-wire steering system has constructional limitations $\delta_{\min } \leq \delta^{e} \leq \delta_{\max }$, it is also considered in the optimization process. The lower and 
upper bounds of the optimal yaw moment is also considered with the following equation:

$$
-m g \mu\left(b_{f}+b_{r}\right) / 2 \leq M_{z}^{e} \leq m g \mu\left(b_{f}+b_{r}\right) / 2 .
$$

Another important constraint to achieving a reasonable solution for the minimization procedure is to guarantee that the signs of the steering angle and yaw moment are equal, i.e. $\operatorname{sign}\left(\delta^{e}\right)=\operatorname{sign}\left(M_{z}^{e}\right)$. Next, the minimization of the total power loss represented by Eq. (8) is evaluated with the constraint given by Eq. (9) and with the constraints on the lower and upper bounds and sign relations regarding the optimal steering angle $\delta^{e}$ and yaw moment $M_{z}^{e}$. The solution of the convex optimization is the optimal steering angle $\delta^{e}$ and yaw moment $M_{z}^{e}$, with which the bus can minimize the power loss due to the cornering resistance. Hence, another variable representing energy efficiency $\rho_{2}^{e}$ is introduced founded on the actual value of the scheduling variable $\rho_{2}$ as follows:

$$
\rho_{2}^{e}=\left\{\begin{array}{lll}
\rho_{2}-\varepsilon, & \text { if } & \left|M_{z}\right| /\left|M_{z}^{e}\right|<1 \\
\rho_{2}+\varepsilon, & \text { if } & \left|M_{z}\right| /\left|M_{z}^{e}\right| \geq 1
\end{array},\right.
$$

where $\varepsilon$ is a scaled value based on the ratio of $M_{z}^{e}$ and $M_{e}$.
The value of $\rho_{2}^{e}$ thus represents the energy optimal control allocation, by which the weighting function $W_{a}$ is set in such way that the LPV controller prescribes a steering angle and yaw moment that minimizes the power loss $P=\left(2 c_{1} \alpha_{1} \delta\right) \dot{\xi}+M_{z} \dot{\psi}$ related to the cornering manoeuvre. However, in safety critical situations due to the failure of an inwheel electric motor, wheel slip, or steering system failure, the reconfiguration is based on the variables $\rho_{2}^{M_{z}}$ and $\rho_{2}^{\delta}$. The strategy of the hierarchical control along with the scheduling variable selection will be presented in the following section.

\section{IMPLEMENTATION OF THE CONTROL SYSTEM}

The energy optimal and fault tolerant reconfigurable controller design of the in-wheel bus is implemented in a hierarchical structure. The multi-layer layout of the proposed control scheme is shown in Fig. 3.

In the first layer, the high-level LPV controller calculates the inputs of the FWIA bus based on the driver reference signals, the measured bus signals regarding the velocity and yaw rate and the value of scheduling variable $\rho_{2}$. Note that $\rho_{2}$ is derived from the energy optimal and fault-tolerant methods. For this purpose, a decision logic has been created with giving priority to the safety of the in-wheel bus. Hence, $\rho_{2}$ is defined as follows:

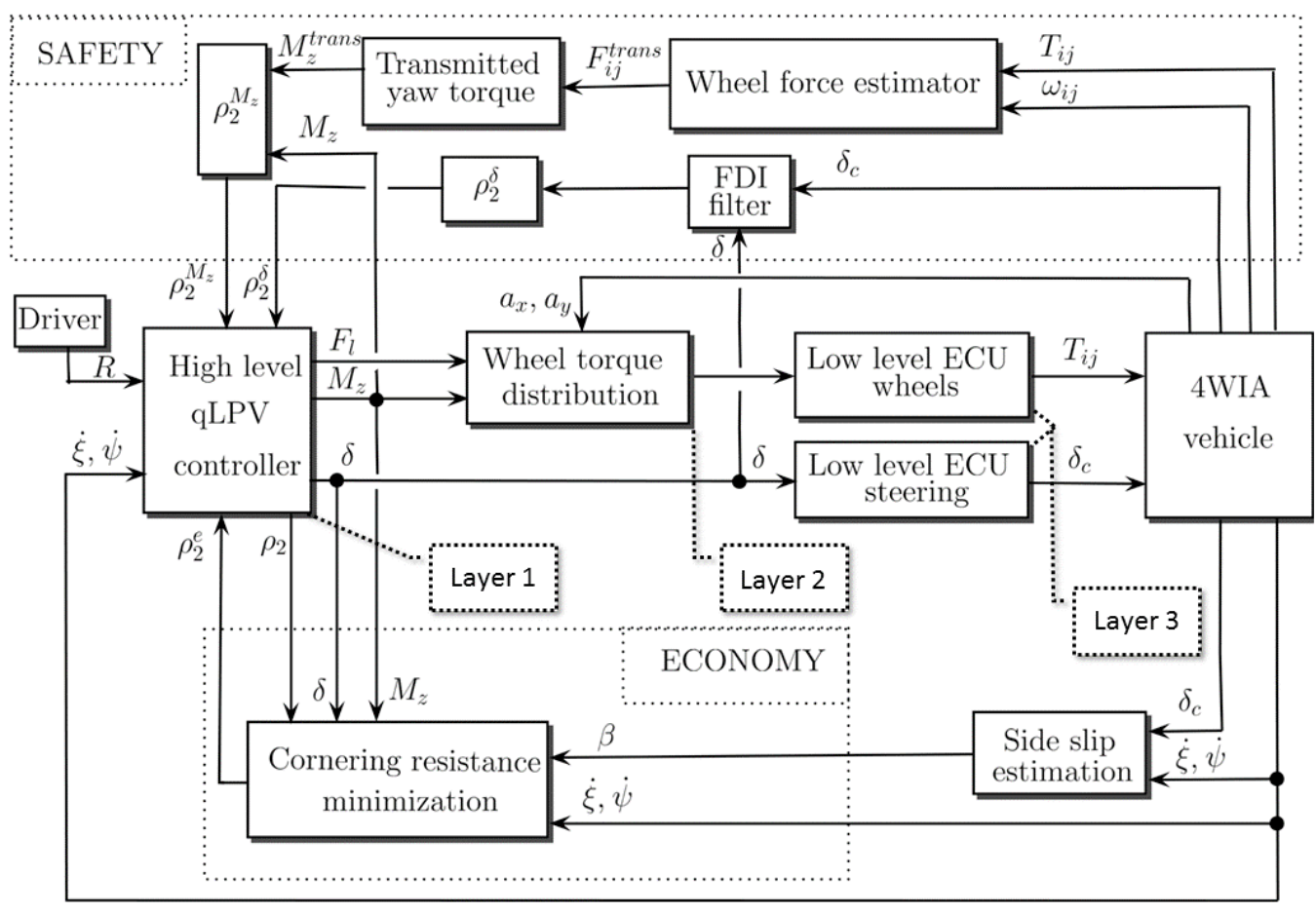

Fig. 3. Hierarchical structure of the reconfigurable control system 


$$
\rho_{2}=\left\{\begin{array}{cccc}
\rho_{2}^{e}, & \text { if } & \rho_{2}^{e}>\rho_{2}^{M_{z}} \quad \text { and } & \rho_{2}^{\delta}=1 \\
\rho_{2}^{M_{z}}, & \text { if } & \rho_{2}^{e} \leq \rho_{2}^{M_{z}} \quad \text { and } & \rho_{2}^{\delta}=1 . \\
\rho_{2}^{\delta}, & \text { if } \quad \rho_{2}^{\delta}=0
\end{array}\right.
$$

Note, that a first-order proportional filter and a hysteresis component are also utilized in order to smooth the value of $\rho_{2}$ and avoid chattering between controllers. The output of the first layer is the highlevel control signals, i.e. the longitudinal force $F_{l}$, the yaw moment $M_{z}$ and the steering angle $\delta$.

The task of the second layer is to distribute the control signals of the LPV controller between the actuators of the in-wheel bus, i.e. the steering system and the in-wheel motors. Since the steering angle is straightforwardly applied, the main objective of the dynamic allocation process is to define the appropriate drive and brake torques for the in-wheel motors based on the high-level control signal $M_{z}$ and $F_{l}$. The dynamic allocation method is implemented in order to define the necessary wheel forces $F_{i j}$ generated by the in-wheel motors. The realized yaw moment of the bus can be written as:

$$
\Delta M_{z}=\left(-F_{f L}+F_{f R}\right) \cdot \frac{b_{f}}{2}+\left(-F_{r L}+F_{r R}\right) \cdot \frac{b_{r}}{2} .
$$

Here the optimization criterion is to minimize the difference between the desired yaw moment $M_{z}$ and the generated yaw moment $\Delta M_{z}$, i.e. function $f_{\text {opt }}$ defined as follows:

$$
f_{\text {opt }}=M_{z}-\Delta M_{z}
$$

In the allocation process, the bus roll and pitch dynamics are also considered in order to avoid the skidding of the wheels during critical bus manoeuvres. Given the longitudinal and lateral accelerations of the bus $a_{x}$ and $a_{y}$ can be measured by low-cost sensors like accelerometers, it is possible to calculate each wheel load given by [19] as follows:

$$
F_{z, i j}=m\left(\frac{l_{[1 ; 2]} g}{L} \pm \frac{h a_{x}}{L}\right) \cdot\left(0.5 \pm \frac{h a_{y}}{b_{[f ; r]} g}\right),
$$

where $L=l_{1}+l_{2}$ is the wheelbase, $h$ is the height of the mass centre. For the calculation of the front wheel loads $(i=f) l_{2}$ and $b_{f}$ is used with a negative sign in the first bracket, while for the rear wheel loads $(i=r) l_{1}$ and $b_{r}$ is used with a positive sign in the first bracket. Note that the left wheel loads $(j=L)$ are given with a negative sign in the second bracket, while the right wheel loads $(j=R)$ with a positive sign.
Thus, the minimal and maximal longitudinal forces for the wheels of the FWIA bus can be expressed as:

$$
F_{i j}^{\max }=\mu F_{z, i j}, \quad F_{i j}^{\min }=-\mu F_{z, i j},
$$

where $\mu$ is the road friction, which is possible to estimate as proposed by [20] and [21]. In order to perform velocity tracking of the FWIA bus, the sum of the wheel forces must be equal to the longitudinal force $F_{l}$ given by the high-level controller. Therefore, the following constraint must also be fulfilled:

$$
\sum F_{i j}-F_{l}=0 \text {. }
$$

Hence, the control allocation leads to a constrained optimization problem, in which the objective function $f_{\text {opt }}=M_{z}-\Delta M_{z}$ introduced in Eq. (14) must be minimized with the constraint of lower and upper bounds given by Eq. (16) and the nonlinear constraint given by Eq. (17). The solution of the optimization is the wheel forces $F_{i j}$, which are transformed into the corresponding in-wheel motor torques $T_{i j}=F_{i j} \cdot R_{e f f}$.

Finally, the third layer contains the specific lowlevel controllers of the steer-by-wire steering system and the four electric in-wheel motors of the bus, transforming the control signals into real physical parameters. Here, the steering is modelled as a first order system as discussed in [22]. Considering the much faster torque response of the in-wheel motors compared to the dynamic response of the wheels, it can be modelled as a second-order system (see [23]) given by the following transfer function: $T_{i j}^{m}(s)=\frac{T_{i j}(s)(1+\eta)}{1+2 \zeta s+2 \zeta^{2} s^{2}}$, where $T_{i j}$ are the required torques, $T_{i j}^{m}$ are the real output torques of the motors, while $\zeta$ and $\eta$ are design parameters considering the response time and steady state error of the electric motors. These parameters depend on the complex structure of the in-wheel electric motor including the inner controller and can be identified by measurements.

\section{SIMULATION RESULTS}

Simulations with the in-wheel electric bus have been performed in a software-in-the-loop (SIL) environment shown in Fig. 4. The upper part represents the electric in-wheel driven bus using a TruckSim/Simulink simulation environment, while the lower part represents the control system with dSPACE MicroAutoBox II on which the proposed control algorithm is running and serving as a fast 
function prototyping electronic control unit (ECU). Note, that the reference signals for the controller and the input signals for the bus are transferred via controller area network (CAN) communication. The aim of the SIL environment is to perform simulation tests for tuning the proposed controller on a platform that can be converted into a hardware-in-the-loop (HIL) system by attaching dSPACE MicroAutoBox II to the CAN system of a real vehicle. By this means, prototype testing can be evaluated with the controller tuned in TruckSim/Simulink environment.

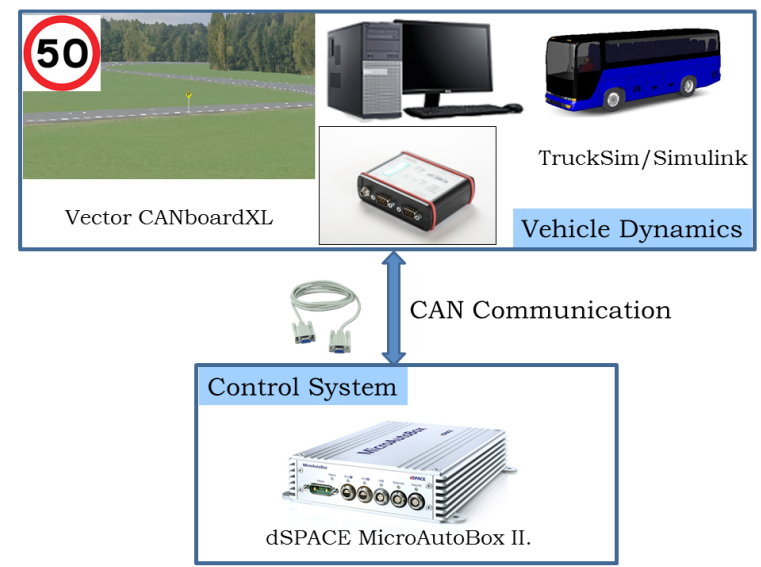

Fig. 4. SIL environment

The simulation vehicle selected in TruckSim is a two-axle bus equipped with four in-wheel motors and a steer-by-wire steering system. In the simulation, the in-wheel bus is assumed to be driven by a driver who must follow the trajectory of an S-turn shown in Fig. 5, while the bus velocity is set to a constant $50 \mathrm{~km} / \mathrm{h}$. The reference yaw rate for the bus to follow shown in Fig. 6 is generated by the driver operating the steering wheel. It is assumed, that the yaw rate, longitudinal and lateral accelerations of the FWIA bus can be measured with low-cost inertial sensors and accelerometers, as well as wheel speeds.

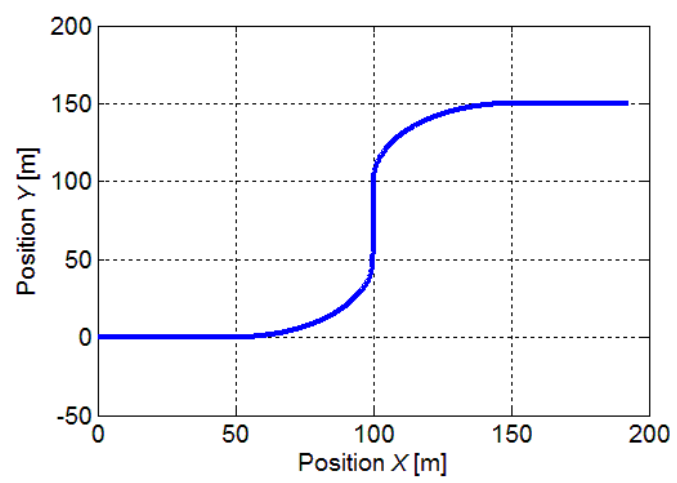

Fig. 5. Geometry of the S-turn

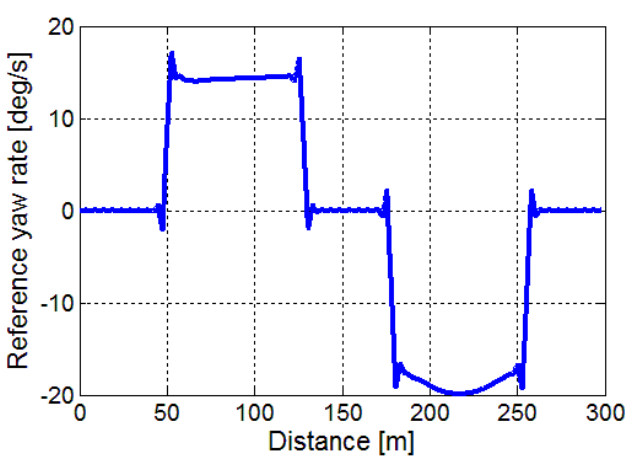

Fig. 6. Reference yaw rate

In the presented simulation case, the effect of an in-wheel motor fault is analysed by comparing the results of the simulation with one evaluated without any fault event.
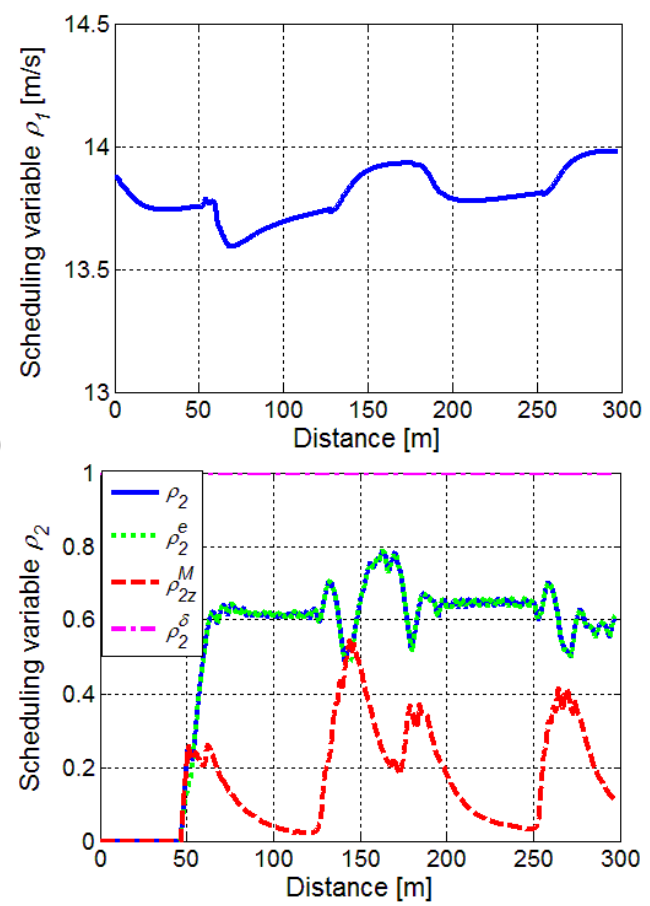

Fig. 7. Scheduling variables without fault; a) $\rho_{1}$ and b) $\rho_{2}$

The scheduling variables of the high-level controller without a fault event are shown in Fig. 7. It is well demonstrated, that the first scheduling variable $\rho_{1}$ shown in Fig. 7a corresponding to the bus velocity remains even in both corners while scheduling variable $\rho_{2}$ responsible for the high-level control allocation is equal to that given by the cornering resistance minimization method, as demonstrated in Fig. $7 b$.

In the case of a faulty in-wheel motor, scheduling variable $\rho_{1}$ becomes more uneven as shown in Fig. 8a. 
The value of the scheduling variable $\rho_{2}$ changes significantly in the second corner, as shown in Fig. 8b. Since the designed yaw moment cannot be put on the road as a result of the faulty in-wheel motor, the value of $\rho_{2}^{M_{z}}$ increases and exceeds the value of $\rho_{2}^{\delta}$ given by the energy optimization method. Hence, the decision logic detailed selects the former as the scheduling variable $\rho_{2}$.

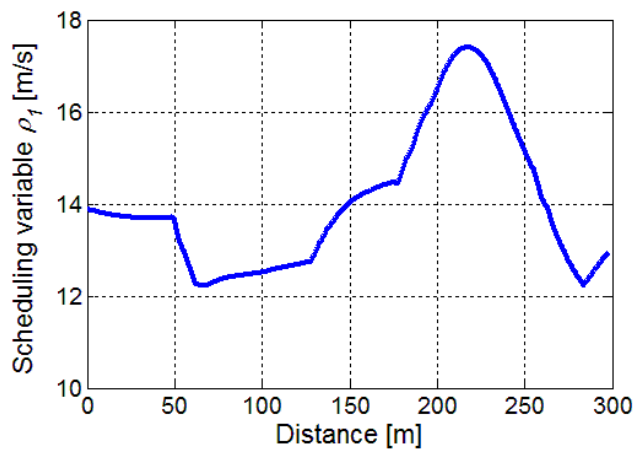

a)

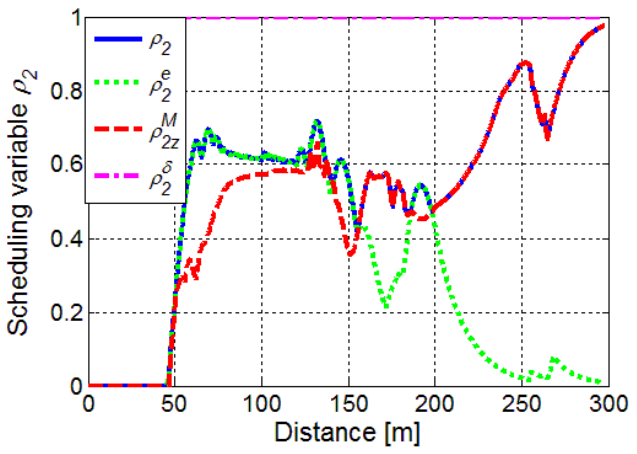

Fig. 8. Scheduling variables with fault; a) $\rho_{1}$ and b) $\rho_{2}$

The prescribed high-level control signals of the LPV controller for both cases are shown in Fig. 9. The operation of the high-level reconfiguration strategy is well demonstrated by observing Figs. $9 \mathrm{~b}$ and c. Without a fault event, the value of $\rho_{2}$ corresponding to the energy optimal cornering yields an effective combination of steering and yaw moment generation. In the case of an in-wheel motor failure and the increased value of $\rho_{2}$, the LPV controller of the FWIA bus prescribes a bigger steering angle for the bus in comparison to the normal case without a fault event.

As a result of the dynamic wheel-torque allocation method, the in-wheel motors generate differential torque considering the bus pitch and roll motion, as shown in Fig. 10. It is well demonstrated by observing Fig. 10a that the left and right side motors, without a fault event generate approximately the same amount of differential torque in both corners.

Meanwhile, when the rear right in-wheel motor fails to produce any torque, as shown in Fig. 10b, the

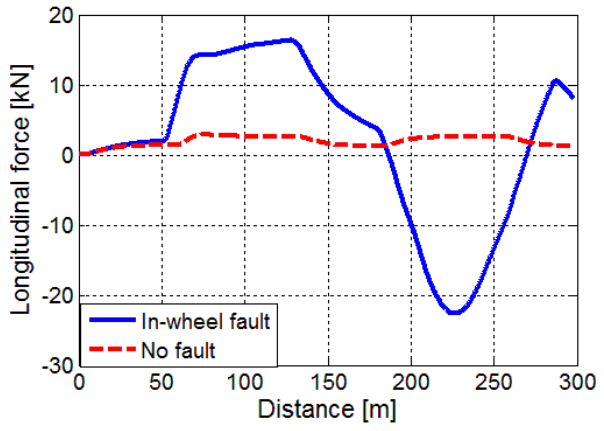

a)

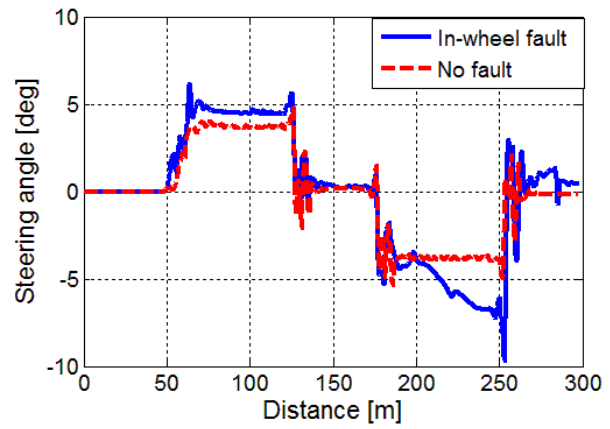

b)

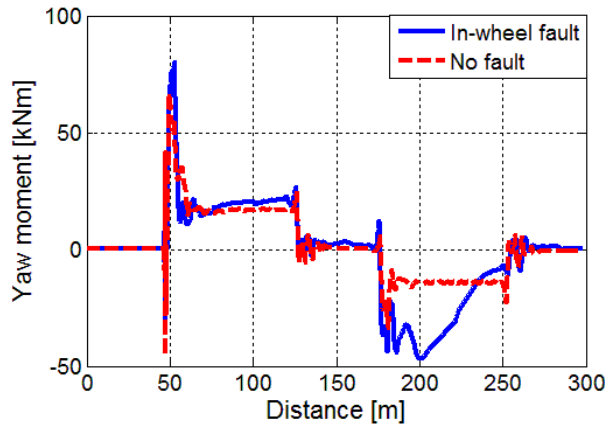

Fig. 9. High-level control signals; a) longitudinal force, b) steering angle; c) yaw moment

differential torque generation of the healthy in-wheel motors are also reduced due to the high-level control reallocation.

The performances of the proposed method are shown in Fig. 11. The velocity error of the FWIA bus does not exceed $1 \mathrm{~km} / \mathrm{h}$ without a fault event as depicted in Fig. 11a. The yaw rate error shown in Fig. $11 \mathrm{~b}$ also remains acceptably small even under a fault event due to the control reconfiguration. It is well demonstrated in Fig. 11c that the cornering energy increases significantly in the case of a fault event when the energy optimal high-level control allocation is overwritten.

\section{CONCLUSIONS}

This paper has proposed an energy optimal and fault tolerant LPV control method for the in-wheel electric 


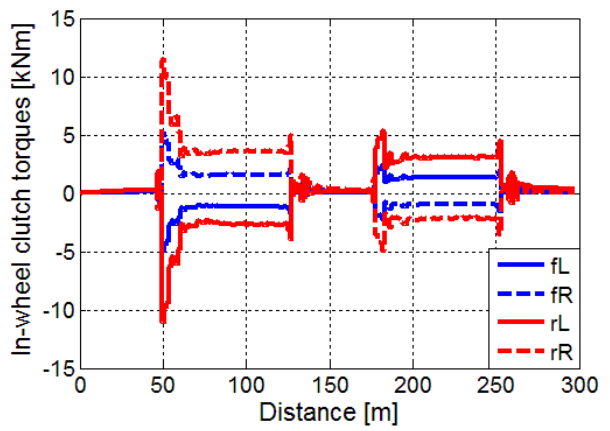

a)

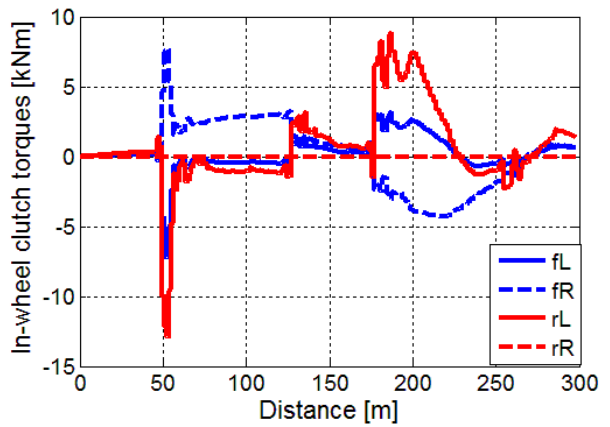

b)

Fig. 10. Hub motor torques; a) wheel torques without fault event, b) wheel torques with motor fault

bus. The proposed strategy is based on a high-level control reconfiguration method, in which the energy efficiency and safety of the FWIA bus are both considered. The operation of the proposed trajectory following control method has been tested in an SIL environment demonstrating the capability of the proposed method.

\section{ACKNOWLEDGMENT}

The research was supported by the National Research, Development and Innovation Fund through the project "SEPPAC: Safety and Economic Platform for Partially Automated Commercial vehicles" (VKSZ 14-1-20150125). This paper was partially supported by the János Bolyai Research Scholarship of the Hungarian Academy of Sciences.

\section{REFERENCES}

[1] Wu, F.-K., Yeh, T.-J., Huang, C.-F. (2013). Motor control and torque coordination of an electric vehicle actuated by two in-wheel motors. Mechatronics, vol. 23, no.1, p.46-60, D0I:10.1016/j.mechatronics.2012.10.008.

[2] Shuai, Z., Zhang, H., Wang, J., Li, J., Ouyang, M. (2013). Lateral motion control for four-wheel-independent-drive electric vehicles using optimal torque allocation and dynamic message priority scheduling. Control Engineering Practice, vol. 24, p. 55-66, D0I:10.1016/J.conengprac.2013.11.012.
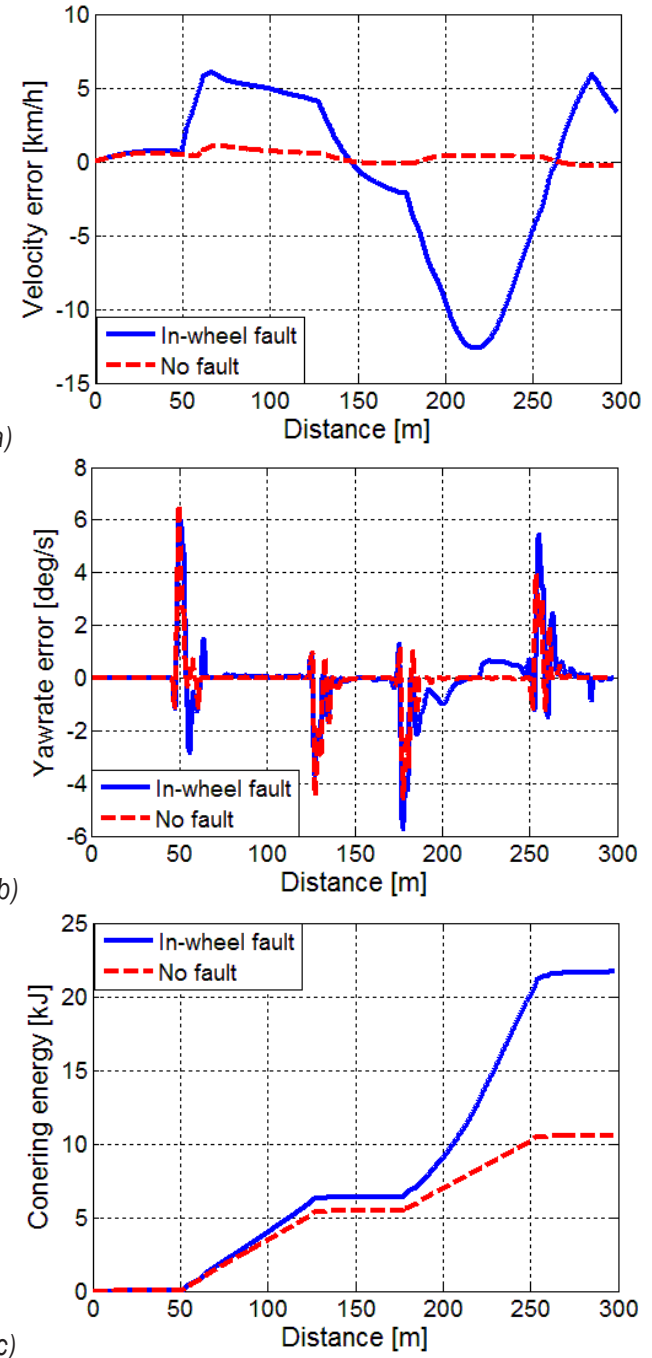

Fig. 11. Performances of the scenarios; a) velocity error, b) yaw rate error, c) cornering energy

[3] de Castro, R., Araújo, R.E., Tanelli, M., Savaresi, S.M., Freitas, D. (2012). Torque blending and wheel slip control in EVs with in-wheel motors. Vehicle System Dynamics, vol. 50, p. 71-94, DOl:10.1080/00423114.2012.666357.

[4] Lin, C., Xu, Z. (2015). Wheel torque distribution of four-wheeldrive electric vehicles based on multi-objective optimization. Energies, vol. 8, no. 5, p. 3815-3831, D0l:10.3390/ en8053815.

[5] Wang, R., Chen, Y., Feng, D., Huang, X., Wang, J. (2011). Development and performance characterization of an electric ground vehicle with independently actuated in-wheel motors. Journal of Power Sources, vol. 196, p. 3962-3971, D0I:10.1016/j.jpowsour.2010.11.160.

[6] Ringdorfer, M., Horn, M. (2011). Development of a wheel slip actuator controller for electric vehicles using energy recuperation and hydraulic brake control. IEEE International 
Conference on Control Applications, Denver, p. 313-318, D0I:10.1109/CCA.2011.6044472.

[7] Li, B., Du, H., Li, W. (2016). Fault-tolerant control of electric vehicles with in-wheel motors using actuator-grouping sliding mode controllers. Mechanical Systems and Signal Processing, vol. 72-73, p. 462-485, DOl:10.1016/j.ymssp.2015.11.020.

[8] Leng, B., Xiong, L., Jin, C., Liu, J. et al. (2015). Differential Drive Assisted Steering Control for an In-wheel Motor Electric Vehicle. SAE International Journal of Passenger CarsElectronic and Electrical Systems, vol. 8, no. 2, p. 433-441, D0I:10.4271/2015-01-1599.

[9] Jing, H., Wang, R., Chadli, M., Hu C., Yan, F., Li, C. (2015). Faulttolerant control of four-wheel independently actuated electric vehicles with active steering systems. 9th IFAC Symposium on Fault Detection, Supervision and Safety for Technical Processes, vol. 48, no. 21, p. 1165-1172, D0l:10.1016/j. ifacol.2015.09.684.

[10] Gáspár, P., Bokor, J., Mihály, A., Szabó, Z. Fülep, T., Szauter F. (2015). Robust reconfigurable control for in-wheel electric vehicles. 9th IFAC Symposium on Fault Detection, Supervision and Safety for Technical Processes, vol. 48, no. 21, p. 36-41, D0I:10.1016/j.ifacol.2015.09.501.

[11] Rajamani, R. (2005). Vehicle Dynamics and Control. Springer, New York, Dol:10.1007/978-1-4614-1433-9.

[12] Bokor, J., Balas, G. (2005). Linear parameter varying systems: A geometric theory and applications. IFAC Proceedings Volumes, vol. 38, no. 1, p. 12-22, D0I:10.3182/20050703-6cz-1902.00003.

[13] Packard, A., Balas, G. (1997). Theory and application of linear parameter varying control techniques. American Control Conference, Workshop I, Albuquerque.

[14] Hu, J.-S., Yin, D. (2011). MTTE-based motion stabilization control for in-wheel motor electric vehicles. Proceedings of SICE Annual Conference, Tokyo, p. 312-317.
[15] Pacejka, H.B. (2004). Tyre and Vehicle Dynamics. Elsevier Butterworth-Heinemann, Oxford.

[16] Im, J.S., Ozaki, F., Yeu, T.K. , Kawaji, S. (2009). Model-based fault detection and isolation in steer-by-wire vehicle using sliding mode observer. Journal of Mechanical Science and Technology, vol. 23, no. 8, p. 1991-1999, Dol:10.1007/ s12206-009-0357-9.

[17] Sumiya, H., Fujimoto, H. (2010). Range extension control system for electric vehicle with active front steering and driving/braking force distribution on curving road. 36th Annual Conference on IEEE Industrial Electronics Society, p. 23522357, DOI:10.1109/IECON.2010.5674927.

[18] Ryu, J., Rossetter, E.J., Gerdes J.C. (2002). Vehicle sideslip and roll parameter estimation using GPS. 6th International Symposium on Advanced Vehicle Control, Hiroshima.

[19] Kiencke, U., Nielsen, L. (2005). Automotive Control Systems. Springer Verlag, Berlin, Heidelberg, D0l:10.1007/b137654.

[20] Gustafsson, F. (1997). Slip-based tire-road friction estimation. Automatica, vol. 33, no. 6, p. 1087-1099, Dol:10.1016/ S0005-1098(97)00003-4.

[21] Li, K., Misener, J. A., Hedrick, K. (2007). On board road condition monitoring system using slip-based tyre road friction estimation and wheel speed signal analysis. Automatica, vol. 221, p. 129-146, D0l:10.1243/1464419jmbd60.

[22] Takanori, F., Shogo, M., Kenji, M., Norihiko, A., Koichi, 0. (2004). Active steering systems based on model reference adaptive nonlinear control. Vehicle System Dynamics, vol. 42, no. 5, p. 301-318, D0I:10.1080/0042311042000266739.

[23] Tahami, F., Kazemi, R., Farhanghi, S. (2003). A novel driver assist stability system for all-wheel-drive electric vehicles. IEEE Transactions on Vehicular Technology, vol. 52, no. 3, p. 683692, D0I:10.1109/TVT.2003.811087. 